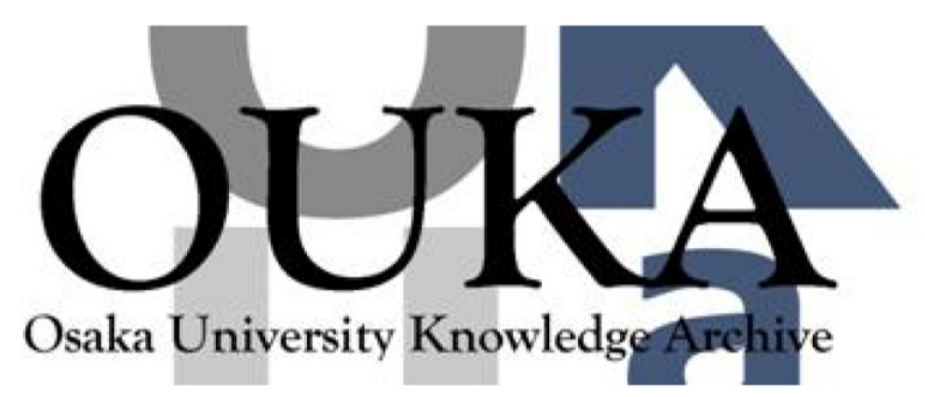

\begin{tabular}{|c|l|}
\hline Title & $\begin{array}{l}\text { Diabetes mellitus itself increases cardio- } \\
\text { cerebrovascular risk and renal complications in } \\
\text { primary aldosteronism }\end{array}$ \\
\hline Author(s) & $\begin{array}{l}\text { Saiki, Aya; Otsuki, Michio; Tamada, Daisuke et } \\
\text { al. }\end{array}$ \\
\hline Citation & $\begin{array}{l}\text { Journal of Clinical Endocrinology and } \\
\text { Metabolism. 105(7) p. e2531-p. e2537 }\end{array}$ \\
\hline Issue Date & $2020-07$ \\
\hline oaire:version & AM \\
\hline URL & https://hdl. handle. net/11094/78291 \\
\hline rights & \\
\hline Note & \\
\hline
\end{tabular}

Osaka University Knowledge Archive : OUKA

https://ir. Library. osaka-u. ac. jp/

Osaka University 


\title{
Diabetes Mellitus Itself Increases Cardio- Cerebrovascular Risk and Renal Complications in Primary Aldosteronism
}

\author{
Aya Saiki, ${ }^{1}$ Michio Otsuki, ${ }^{1}$ Daisuke Tamada, ${ }^{1}$ Tetsuhiro Kitamura, ${ }^{1}$ \\ lichiro Shimomura, ${ }^{1}$ Isao Kurihara, ${ }^{2}$ Takamasa Ichijo, ${ }^{3}$ Yoshiyu Takeda, ${ }^{4}$ \\ Takuyuki Katabami, ${ }^{5}$ Mika Tsuiki, ${ }^{6}$ Norio Wada, ${ }^{7}$ Toshihiko Yanase, ${ }^{8}$ \\ Yoshihiro Ogawa, ${ }^{9}$ Junii Kawashima, ${ }^{10}$ Masakatsu Sone, ${ }^{11}$ Nobuya Inagaki, ${ }^{11}$ \\ Takanobu Yoshimoto, ${ }^{12,13}$ Ryuji Okamoto, ${ }^{14}$ Katsutoshi Takahashi, ${ }^{15}$ \\ Hiroki Kobayashi, ${ }^{16}$ Kouichi Tamura, ${ }^{17}$ Kohei Kamemura ${ }^{18}$ Koichi Yamamoto, ${ }^{19}$ \\ Shoichiro Izawa, ${ }^{20}$ Miki Kakutani, ${ }^{21}$ Masanobu Yamada, ${ }^{22}$ Akiyo Tanabe, ${ }^{23}$ and \\ Mitsuhide Naruse ${ }^{6,24}$
}

\begin{abstract}
${ }^{1}$ Department of Metabolic Medicine, Osaka University Graduate School of Medicine, Osaka 565-0871, Japan; ${ }^{2}$ Department of Endocrinology, Metabolism, and Nephrology, School of Medicine, Keio University, Tokyo 160-8582, Japan; ${ }^{3}$ Department of Endocrinology and Metabolism, Saiseikai Yokohamashi Tobu Hospital, Yokohama 239-0012, Japan; ${ }^{4}$ Department of Internal Medicine, Graduate School of Medical Sciences, Kanazawa University, Kanazawa 920-8640, Japan; ${ }^{5}$ Division of Metabolism and Endocrinology, Department of Internal Medicine, St. Marianna University School of Medicine, Yokohama City Seibu Hospital, Yokohama 241-0811, Japan; ${ }^{6}$ Department of Endocrinology and Metabolism, National Hospital Organization Kyoto Medical Center, Kyoto 612-8555, Japan; ${ }^{7}$ Department of Diabetes and Endocrinology, Sapporo City General Hospital, Sapporo 060-8604, Japan; ${ }^{8}$ Muta Hospital, Fukuoka 814-0163, Japan; ${ }^{9}$ Department of Medicine and Bioregulatory Science, Graduate School of Medical Sciences, Kyushu University, Fukuoka 812-8582, Japan; ${ }^{10}$ Department of Metabolic Medicine, Faculty of Life Sciences, Kumamoto University, Kumamoto 860-8556, Japan; ${ }^{11}$ Department of Diabetes, Endocrinology and Nutrition, Graduate School of Medicine, Kyoto University, Kyoto 606-8507, Japan; ${ }^{12}$ Department of Molecular Endocrinology and Metabolism, Tokyo Medical and Dental University, Tokyo 113-8510, Japan; ${ }^{13}$ Department of Diabetes and Endocrinology, Tokyo Metropolitan Hiroo Hospital Tokyo, Tokyo 150-0013, Japan; ${ }^{14}$ Department of Cardiology, Mie University Hospital, Mie 514-8507, Japan; ${ }^{15}$ Division of Metabolism, Showa General Hospital, Tokyo 187-8510, Japan; ${ }^{16}$ Division of Nephrology, Hypertension, and Endocrinology, Nihon University School of Medicine, Tokyo 173-8610, Japan; ${ }^{17}$ Department of Medical Science and Cardiorenal Medicine, Yokohama City University Graduate School of Medicine, Yokohama 236-0004, Japan; ${ }^{18}$ Department of Cardiology, Shinko Hospital, Kobe 651-0072, Japan; ${ }^{19}$ Department of Geriatric and General Medicine, Osaka University Graduate School of Medicine, Osaka 565-0871, Japan; ${ }^{20}$ Department of Endocrinology and Metabolism, Tottori University Hospital, Yonago 683-8504, Japan; ${ }^{21}$ Division of Diabetes, Endocrinology, and Clinical Immunology, Department of Internal Medicine, Hyogo College of Medicine, Nishinomiya 663-8501, Japan; ${ }^{22}$ Department of Medicine and Molecular Science, Gunma University Graduate School of Medicine, Maebashi 371-8511, Japan; ${ }^{23}$ Division of Endocrinology, National Center for Global Health and Medicine, Tokyo 162-8655, Japan; and ${ }^{24}$ Endocrine Center, ljinkai Takeda General Hospital, Kyoto 601-1495, Japan
\end{abstract}

ORCiD numbers: 0000-0001-5947-8621 (M. Otsuki); 0000-0001-8261-2593 (N. Inagaki); 0000-0002-8067-398X (R. Okamoto); 0000-0002-0660-5372 (K. Tamura).

Context: The prevalence of diabetes mellitus (DM) in patients with primary aldosteronism (PA) is higher than in those with essential hypertension and the general population. Although DM is

ISSN Print 0021-972X ISSN Online 1945-7197

Printed in USA

C) Endocrine Society 2020. All rights reserved. For permissions, please e-mail: journals. permissions@oup.com

Received 10 January 2020. Accepted 9 April 2020.

First Published Online 10 April 2020.

Corrected and Typeset 6 May 2020.
Abbreviations: AVS, adrenal vein sampling; CCV, cardio-cerebrovascular; DM, diabetes mellitus; eGFR, estimated glomerular filtration rate; EH, essential hypertension; PA, primary aldosteronism. 
a common major risk factor for cardio-cerebrovascular (CCV) diseases and renal complications, details of its effects in PA have not been demonstrated.

Objective: The aim of this study was to determine the effects of coexistent DM on the risk of CCV events and progression of renal complications in PA patients.

Design: A multi-institutional, cross-sectional study was conducted.

Patients and Methods: PA patients experienced between January 2006 and October 2016 and with available data of CCV events and DM were enrolled from the Japan PA registry of the Japan Primary Aldosteronism Study/Japan Rare Intractable Adrenal Diseases Study $(n=2524)$. CCV events and renal complications were compared between a DM group and a non-DM group by logistic and liner-regression analysis.

Results: DM significantly increased the odds ratio (OR) of CCV events (OR $1.59,95 \%$ Cl: $1.05-$ $2.41)$ and that of proteinuria (OR $2.25,95 \% \mathrm{Cl}: 1.59-3.16)$. DM correlated significantly with declines in estimated glomerular filtration rate $(\beta=.05, P=.02)$.

Conclusions: This the first report to demonstrate the presence of DM as an independent risk factor for CCV events and renal complications, even in PA patients. Management of DM should be considered in addition to the specific treatment of PA. (J Clin Endocrinol Metab 105: e2531-e2539, 2020)

Freeform/Key Words: primary aldosteronism, diabetes mellitus, cardio-cerebrovascular events, proteinuria, eGFR

$\mathbf{P}$ rimary aldosteronism (PA) is a major cause of secondary hypertension that affects $3.2 \%$ to $12.7 \%$ of hypertensive patients (1). PA patients are at higher risks of cardio-cerebrovascular (CCV) events compared to those with essential hypertension (EH), and the increased risk is mainly due to excess aldosterone, which induces not only a rise in blood pressure but also directly damages the myocardium and arterial walls $(2,3)$. Moreover, persistent exposure to excessive aldosterone is known to cause renal fibrosis and vasculopathies (4, $5)$. This explains the high urinary albumin excretion rate in PA patients compared with EH patients (6). On the other hand, in patients with diabetes mellitus (DM), the risks of CCV events and proteinuria are high because of hyperglycemia (7).

The prevalence of DM in PA patients is $8.2 \%$ to $23.0 \%(8-10)$ and is higher than in $\mathrm{EH}$ patients and the general population (11). Though both DM and PA play important roles in the progression of CCV diseases and renal complications, the relationship between DM and these diseases in PA patients has not been evaluated.

The aim of this study was to determine whether DM co-pathology in PA patients increases the risk of CCV events and progression of renal complications.

\section{Methods}

\section{Study design}

This retrospective cross-sectional study was conducted as part of the Japan Primary Aldosteronism Study/Japan Rare Intractable Adrenal Diseases Study (JPAS/JRAS) study including 30 referral centers. Patients with PA who were diagnosed and underwent adrenal vein sampling (AVS) from January 2006 to February 2018 were enrolled in this study. Patients eligible for JPAS were men and women age 20 to 90 years. Patients deemed unsuitable by the investigators were excluded. The clinical characteristics, biochemical findings, and AVS results were collected electronically using the web registry system. System construction, data security, and maintenance of the registered data were outsourced to the EPS Corporation.

The study was conducted using a data set valid as of February 2018. Only patients with data available on clinical course and biochemistry laboratory test results, especially those related to DM and medical history of CCV events, were included. We excluded patients with suspected autonomous cortisol secretion, defined by serum cortisol levels greater than $3 \mu \mathrm{g} / \mathrm{dL}$ after $1 \mathrm{mg}$ dexamethasone.

We defined DM as glycated hemoglobin greater than or equal to $6.5 \%$ (National Glycohemoglobin Standardization Program), or treatment with medications or dietary and exercise therapy specific for DM. Glycated hemoglobin greater than or equal to $6.5 \%$ is considered to represent $\mathrm{DM}$ according to the American Diabetes Association (12) and the Japan Diabetes Society (13). CCV events were defined as myocardial infarction, heart failure (required hospitalization for treatment), cerebral infarction, and cerebral hemorrhage. Proteinuria was defined as,$+ 2+$, and $3+$ protein on urinalyses.

\section{Diagnosis of primary aldosteronism}

The diagnosis of PA was based on the criteria defined by the Japan Endocrine Society (14) and Japan Society of Hypertension (15). PA was diagnosed by positive case detection with a ratio of plasma aldosterone concentration (PAC) (measured in ng/ $\mathrm{dL}$ ) to plasma renin activity (PRA) (measured in $\mathrm{ng} / \mathrm{mL} / \mathrm{h}$ ) of greater than 20 and at least 1 positive result of 4 confirmatory tests, including the captopril-challenge test, the saline-infusion test, the furosemide-upright test, and the oral salt-loading test. Antihypertensive medications were usually changed to calcium channel blockers and/or $\alpha$-adrenergic blockers, as appropriate, until the final diagnosis was established. 


\section{Hormone assays}

PAC and PRA were measured by commercially available kits. PAC was determined by radioimmunoassay in 27 centers and chemiluminescent enzyme immunoassay in 3 centers. PRA was determined by radioimmunoassay in 20 centers and EIA in 9 centers. Plasma active renin concentration was measured by chemiluminescent enzyme immunoassay in one center and the value was used for analysis by converting to PRA.

\section{Statistical analysis}

Data were expressed as median values (first and third quartiles). Differences between parameters of 2 groups were compared by the Wilcoxon test for continuous variables and by chi-square test for categorical variables. Significant variables were included in a multivariable logistic regression model to determine the independent risk factors associated with CCV events and proteinuria. Multiple regression analysis was used for estimated glomerular filtration rate (eGFR). Statistical significance was defined as a significance level of $P$ less than .05 . All statistical analyses were performed using JMP Pro software for Window (ver. 14, SAS Institute).

\section{Results}

\section{Patients}

Patients with PA with available data on CCV events and $\mathrm{DM}(\mathrm{n}=2524$, men, $47.5 \%)$ were enrolled in this study. Table 1 summarizes the clinical characteristics of these patients. The median age was 53 years (range, 45-62 years), median body mass index (BMI) was $24.4 \mathrm{~kg} / \mathrm{m}^{2}$ (range, $21.9-27.3 \mathrm{~kg} / \mathrm{m}^{2}$ ), median systolic blood pressure (SBP) was $140 \mathrm{~mm} \mathrm{Hg}$ (range, 129-152 $\mathrm{mm} \mathrm{Hg}$ ), median diastolic blood pressure (DBP) was $86 \mathrm{~mm} \mathrm{Hg}$ (range, 78-95 mm Hg), median duration of hypertension at the time of PA diagnosis was 5 years (range, 2-12 years), the mean number of antihypertensive classes was 1.0 (range, 1.0-1.0), median PAC was $17.8 \mathrm{ng} / \mathrm{dL}$ (range, 12.6-27.9 ng/dL), median PRA was $0.3 \mathrm{ng} / \mathrm{mL} / \mathrm{h}$ (range, $0.2-0.5 \mathrm{ng} / \mathrm{mL} / \mathrm{h}$ ), and the median plasma aldosterone/plasma renin activity ratio was 55.8 (range, 32.8-114.6). A unilateral subtype was identified in 621 patients $(30.3 \%)$ and bilateral subtype in 1426 patients. Of the total, $26.9 \%$ had dyslipidemia and $41.4 \%$ had hypokalemia. Of the 2524 PA patients, $389(15.4 \%)$ had DM, and $182(7.2 \%)$ CCV events were recorded in these patients, with cerebral infarction being the most common. The incidence of proteinuria was $10.7 \%$. The median eGFR was $77.9 \mathrm{~mL} / \mathrm{min} / 1.73$ $\mathrm{m}^{2}$ (range, 66.4-91.4 $\mathrm{mL} / \mathrm{min} / 1.73 \mathrm{~m}^{2}$ ).

\section{Comparison of clinical characteristic between the diabetes mellitus group and the non-diabetes mellitus group in primary aldosteronism patients}

Table 2 shows the differences between PA patients of the DM group and the non-DM group. As stated

\begin{tabular}{lc}
\hline $\begin{array}{l}\text { Table 1. Clinical characteristics of the } \mathbf{2 5 2 4} \text { patients } \\
\text { with primary aldosteronism }\end{array}$ \\
\hline No. & $\mathbf{2 5 2 4}$ \\
\hline Sex, male & $47.5 \%$ \\
Age, y & $53(45-62)$ \\
Body mass index, $\mathrm{kg} / \mathrm{m}^{2}$ & $24.4(21.9-27.3)$ \\
Systolic blood pressure, $\mathrm{mm} \mathrm{Hg}$ & $140(129-152)$ \\
Diastolic blood pressure, mm Hg & $86(78-95)$ \\
Duration of hypertension, y & $5(2-12)$ \\
No. of antihypertensive drugs & $1.0(1.0-1.0)$ \\
Plasma aldosterone, $\mathrm{ng} / \mathrm{dL}$ & $17.8(12.6-27.9)$ \\
Plasma renin activity, $\mathrm{ng} / \mathrm{mL} / \mathrm{h}$ & $0.3(0.2-0.5)$ \\
ARR, ng/dL/ng/mL/h & $55.8(32.8-114.6)$ \\
Unilateral subtype & $30.3 \%$ \\
Hypokalemia & $41.4 \%$ \\
Serum potassium, mEg/L & $3.8(3.4-4.0)$ \\
Diabetes mellitus & $15.4 \%$ \\
FBS, mg/dL & $98(91-109)$ \\
HbA ${ }_{1}$ NGSP, \% & $5.6(5.3-6.0)$ \\
Dyslipidemia & $26.9 \%$ \\
Current or past smoking & $35.8 \%$ \\
Current or past drinking & $53.7 \%$ \\
CCV events & $7.2 \%$ \\
Myocardial infarction & $0.9 \%$ \\
Cerebral hemorrhage & $2.4 \%$ \\
Cerebral infarction & $4.1 \%$ \\
Heart failure & $0.5 \%$ \\
Proteinuria & $12.1 \%$ \\
eGFR, mL/min/1.73 m ${ }^{2}$ & $77.9(66.4-91.4)$ \\
\hline
\end{tabular}

The values in parentheses are median (first and third quartiles). Unilateral subtype was evaluated by adrenal vein sampling results with adrenocorticotropic hormone (cosyntropin) stimulation if cannulation was successful (selectivity index $>5$ ). The selectivity index was defined as the cortisol concentration in the adrenal vein relative to that in the inferior vena cava. The diagnosis of unilateral PA subtype was defined as a lateralization index greater than 4 . The lateralization index was calculated by that on the nondominant side. Hypokalemia represented serum potassium less than $3.5 \mathrm{mEq} / \mathrm{L}$ at the diagnosis of PA or taking potassium supplementation. Oral potassium was supplemented if hypokalemia was present. eGFR was obtained using the following equation: eGFR $\left(\mathrm{mL} / \mathrm{min} / 1.73 \mathrm{~m}^{2}\right)=194 \times$ serum creatinine $(-1.094) \times$ age $(-0.287) \times 0.739$ (for women). Proteinuria was defined as,$+ 2+$, and $3+$ protein on urinalysis.

Abbreviations: ARR, plasma aldosterone/plasma renin activity ratio; CCV events, cardio-cerebrovascular events; eGFR, estimated glomerular filtration rate; FBS, fasting blood sugar; $\mathrm{HbA}_{1 c^{\prime}}$ hemoglobin $\mathrm{A}_{1 \mathrm{c}^{\prime}}$; NGSP, National Glycohemoglobin Standardization Program; PA, primary aldosteronism.

earlier, 389 (15.4\%) of the 2524 PA patients had DM. Compared with the non-DM group, the DM group included significantly more older ( 59 vs 53 years, $P<.01$ ), obese (BMI, 25.9 vs $24.1 \mathrm{~kg} / \mathrm{m}^{2}, P<.01$ ), smoking (44.3 vs $34.3 \%, P<.01)$ men $(60.8$ vs $45.2 \%, P<.01)$ with a longer duration of hypertension (10 vs 5 years, $P<.01)$, taking more antihypertensive drugs $(1.0[1.0-$ $2.0]$ vs 1.0 [1.0-1.0], $P<.01)$, and a higher incidence of dyslipidemia ( 45.0 vs $23.6 \%, P<.01$ ). There were no significant differences in PAC, PRA, and incidence of hypokalemia between the 2 groups. PA subtype classified by AVS was similar (unilateral, 29.8 vs $30.4 \%$, $P=.84)$ in both groups. 


\begin{tabular}{|c|c|c|c|}
\hline & DM $(n=389)$ & Non-DM $(n=2135)$ & $P$ \\
\hline Sex, male & $60.7 \%$ & $45.2 \%$ & $<.01$ \\
\hline Age, y & $59(51-65)$ & $53(44-61)$ & $<.01$ \\
\hline Body mass index, $\mathrm{kg} / \mathrm{m}^{2}$ & $25.9(23.1-29.2)$ & $24.1(21.7-26.9)$ & $<.01$ \\
\hline Systolic blood pressure, mm Hg & $141(129-154)$ & $140(129-152)$ & .19 \\
\hline Diastolic blood pressure, $\mathrm{mm} \mathrm{Hg}$ & $85(76-93)$ & $87(78-95)$ & $<.01$ \\
\hline Duration of hypertension, y & $10(2-19)$ & $5(2-11)$ & $<.01$ \\
\hline Vo. of antihypertensive drugs & $1.0(1.0-2.0)$ & $1.0(1.0-1.0)$ & $<.01$ \\
\hline Plasma aldosterone, ng/dL & $17.2(12.5-26.8)$ & $17.9(12.6-28.3)$ & .24 \\
\hline lasma renin activity, ng/mL/h & $0.3(0.2-0.5)$ & $0.3(0.2-0.5)$ & .38 \\
\hline ARR, ng/dL/ng/mL/h & $52.6(32.4-97.7)$ & $56.3(32.8-118.0)$ & .16 \\
\hline Jnilateral subtype & $29.8 \%$ & $30.4 \%$ & .84 \\
\hline Hypokalemia & $39.8 \%$ & $41.7 \%$ & .51 \\
\hline Serum potassium, mEq/L & $3.8(3.4-4.1)$ & $3.8(3.4-4.0)$ & .08 \\
\hline BS, $\mathrm{mg} / \mathrm{dL}$ & $128(110-154)$ & $96(90-104)$ & $<.01$ \\
\hline $\mathrm{HbA}_{1 c}$ NGSP, \% & $6.8(6.4-7.8)$ & $5.5(5.3-5.8)$ & $<.01$ \\
\hline Dyslipidemia & $45.0 \%$ & $23.6 \%$ & $<.01$ \\
\hline Current or past smoking & $44.3 \%$ & $34.3 \%$ & $<.01$ \\
\hline Current or past drinking & $51.0 \%$ & $54.2 \%$ & \\
\hline CCV events & $13.9 \%$ & $6.0 \%$ & $<.01$ \\
\hline Myocardial infarction & $2.6 \%$ & $0.6 \%$ & $<.01$ \\
\hline Cerebral hemorrhage & $3.1 \%$ & $2.3 \%$ & .35 \\
\hline Cerebral infarction & $8.2 \%$ & $3.4 \%$ & $<.01$ \\
\hline Heart failure & $1.5 \%$ & $0.3 \%$ & $<.01$ \\
\hline Proteinuria & $24.8 \%$ & $9.6 \%$ & $<.01$ \\
\hline eGFR, $\mathrm{mL} / \mathrm{min} / 1.73 \mathrm{~m}^{2}$ & $76.8(63.5-91.0)$ & $78.4(66.9-91.5)$ & $<.05$ \\
\hline
\end{tabular}

The values in parentheses are median (first and third quartiles). $P$ values for differences between the DM and non-DM group (sex, current or past smoking, current or past drinking, unilateral subtype, hypokalemia, dyslipidemia, proteinuria, and CCV events) by the chi-square test, for others by the Wilcoxon test.

Abbreviations: ARR, plasma aldosterone/plasma renin activity ratio; CCV events, cardio-cerebrovascular events; DM, diabetes mellitus; eGFR, estimated glomerular filtration rate; FBS, fast blood sugar; $\mathrm{HbA}_{1 c^{\prime}}$, hemoglobin $\mathrm{A}_{1 c^{\prime}}$ NGSP, National Glycohemoglobin Standardization Program.

The rate of CCV events was significantly higher in the DM group ( 13.9 vs $6.0 \%, P<.01)$, especially for myocardial infarction ( 2.6 vs $0.6 \%, P<.01)$, cerebral infarction $(8.2$ vs $3.4 \%, P<.01)$, and heart failure $(1.5$ vs $0.3 \%, P<.01)$. With regard to renal complications, the incidence of proteinuria was significantly higher $(24.8$ vs $9.6 \%, P<.01)$ and eGFR was significantly lower $\left(76.8\right.$ vs $\left.78.4 \mathrm{~mL} / \mathrm{min} / 1.73 \mathrm{~m}^{2}, P<.05\right)$ in the DM group than in the non-DM group.

\section{Logistic regression analysis for cerebro- cardiovascular events and renal complications}

Logistic regression analysis was performed to investigate the relation of sex, age, BMI, SBP, duration of hypertension, dyslipidemia, smoking, and drinking with CCV events and renal complications. In addition to male sex, older age, long duration of hypertension, dyslipidemia, and smoking, DM (odds ratio [OR] 1.59, 95\% CI: 1.05-2.41) significantly increased, whereas drinking decreased the OR of CCV events (Table 3). With regard to renal complications, DM (OR 2.25, 95\% CI: 1.59-3.16), in addition to other parameters, such as male sex, obesity, high SBP, and long duration of hypertension, significantly increased the OR of proteinuria
(Table 4). Multiple regression analysis identified DM $(\beta=.05, P=.02)$, male sex, old age, and long duration of hypertension to be significant correlates with the decline in eGFR (Table 5).

In patients of the nonproteinuria group, eGFR correlated significantly with $\mathrm{DM}(\beta=.09, P<.01)$, whereas no such correlation was found in the proteinuria group $(\beta=-.01, P=.85)$.

\section{Discussion}

PA and DM are both well-known important risk factors for CCV events and renal complications. In this study, we demonstrated that DM was an independent risk factor for CCV events and renal complications in PA patients. With regards to PA subtypes, the incidences of CCV events and proteinuria both in unilateral and bilateral subtypes with DM were higher compared with those without DM, respectively (CCV events in unilateral; DM group vs non-DM group $21.3 \%$ vs $8.2 \%, P<.01$, proteinuria in unilateral; DM group vs non-DM group $36.7 \%$ vs $17.3 \%, P<.01$, CCV events in bilateral; DM group vs non-DM group $10.0 \%$ vs $4.8 \%, P<.01$, proteinuria in bilateral; DM group vs 


\section{Table 3. Risk factors for cardio-cerebrovascular events}

\begin{tabular}{|c|c|c|c|c|}
\hline Variable & \multicolumn{2}{|c|}{ Univariate analysis } & \multicolumn{2}{|c|}{ Multivariable analysis } \\
\hline Diabetes mellitus & $2.53(1.80-3.55)$ & $<.01$ & $1.59(1.05-2.41)$ & .03 \\
\hline Sex, male & $2.09(1.53-2.86)$ & $<.01$ & $1.98(1.29-3.04)$ & $<.01$ \\
\hline Age, +10 y & $1.70(1.47-1.98)$ & $<.01$ & $1.40(1.15-1.71)$ & $<.01$ \\
\hline Body mass index,$+5 \mathrm{~kg} / \mathrm{m}^{2}$ & $1.09(0.91-1.31)$ & .33 & $0.96(0.76-1.22)$ & .74 \\
\hline Systolic blood pressure, +10 mm Hg & $1.02(0.94-1.11)$ & .56 & $1.03(0.94-1.14)$ & .54 \\
\hline Dyslipidemia & $2.34(1.72-3.18)$ & $<.01$ & $1.61(1.11-2.34)$ & .01 \\
\hline Current or past smoking & $1.73(1.25-2.39)$ & $<.01$ & $1.58(1.06-2.34)$ & .02 \\
\hline Current or past drinking & $0.71(0.52-0.99)$ & .04 & $0.50(0.34-0.74)$ & $<.01$ \\
\hline
\end{tabular}

Abbreviation: OR, odds ratio.

\section{Table 4. Risk factors for proteinuria}

\begin{tabular}{|c|c|c|c|c|}
\hline Variable & \multicolumn{2}{|c|}{ Univariate analysis } & \multicolumn{2}{|c|}{ Multivariable analysis } \\
\hline Diabetes mellitus & $3.09(2.34-4.09)$ & $<.01$ & $2.25(1.59-3.16)$ & $<.01$ \\
\hline Sex, male & $3.26(2.47-4.31)$ & $<.01$ & $2.73(1.91-3.92)$ & $<.01$ \\
\hline Age, +10 y & $1.20(1.07-1.34)$ & $<.01$ & $1.02(0.88-1.20)$ & .77 \\
\hline Body mass index,$+5 \mathrm{~kg} / \mathrm{m}^{2}$ & $1.64(1.42-1.89)$ & $<.01$ & $1.31(1.10-1.57)$ & $<.01$ \\
\hline Systolic blood pressure, $+10 \mathrm{~mm} \mathrm{Hg}$ & $1.20(1.13-1.29)$ & $<.01$ & $1.20(1.11-1.30)$ & $<.01$ \\
\hline Current or past smoking & $1.51(1.15-1.98)$ & $<.01$ & $0.97(0.70-1.34)$ & .87 \\
\hline Current or past drinking & $1.23(0.94-1.62)$ & .13 & $0.92(0.67-1.28)$ & .63 \\
\hline
\end{tabular}

Abbreviation: OR, odds ratio.

Table 5. Results of multiple linear regression analysis for the association of estimated glomerular filtration rate with various factors

\begin{tabular}{lcrr}
\hline & \multicolumn{2}{c}{ Univariate analysis } & \multicolumn{2}{c}{ Multivariable analysis } \\
\cline { 2 - 4 } Variable & $\beta$ & $\boldsymbol{P}$ & $\beta$ \\
\hline Diabetes mellitus & -.05 & .01 & .05 \\
Sex, male & -.12 & $<.01$ & -0.12 \\
Age & -.43 & $<.01$ & -0.41 \\
Body mass index & -.01 & .77 & -0.03 \\
Systolic blood pressure & -.03 & .12 & -0.03 \\
Duration of hypertension & -.24 & $<.01$ & -0.07 \\
Dyslipidemia & -.08 & $<.01$ & -0.00004 \\
Current or past smoking & -.03 & .11 & -0.01 \\
Current or past drinking & -.0003 & .99 & 0.03 \\
\hline
\end{tabular}

non-DM group $18.7 \%$ vs $6.4 \%, P<.01$ ). These results suggest that DM itself plays an important role in the progression of CCV diseases and proteinuria in spite of PA subtypes.

A recent meta-analysis has shown that PA patients are at significantly higher risk of CCV events compared with $\mathrm{EH}$ patients (stroke OR 2.58 [95\% CI: 1.93-3.45], coronary heart disease OR 1.77 [95\% CI: 1.10-2.83], heart failure OR 2.05 (95\% CI: 1.11-3.78]) (2). Moreover, PA patients have a higher urine albumin excretion rate than $\mathrm{EH}$ patients (6). It is known that adrenalectomy and treatment with mineralocorticoid receptor antagonists are effective treatments that reduce CCV event risk $(16,17)$ and proteinuria $(18)$ in PA patients. Therefore, early diagnosis and treatment of PA should prevent these risks.

The prevalence of DM is higher in PA than EH and the general population (11). In the present study, the prevalence of DM in PA patients was $15.4 \%$, which is similar to those of previous studies (range, 8.2-23.0\%) $(8-10)$. In the former JPAS study whose patients with autonomous cortisol secretion were included, the 
prevalence of DM in PA patients was higher than in the present study $(21.6 \%$ vs $15.4 \%)$ (19). Hypokalemia and hyperaldosteronemia are known for the risk factors of DM in PA patients. However, there were no significant differences in the incidence of hypokalemia and PAC levels between the DM group and the non-DM group in our study. The same results have been reported in previous studies $(8,20)$. These results suggested that aging, high BMI, and lifestyle habits (21) have a larger contribution to the onset of glucose intolerance compared with hypokalemia and hyperaldosteronemia.

Current or past drinking decreased the OR for CCV events (OR 0.50, 95\% CI: 0.34-0.74) in our study. Although we do not have any data about alcohol consumption, Saito et al (22) reported that the association of alcohol consumption with mortality due to heart disease and cerebrovascular disease was examined in the Japanese population. Light to moderate alcohol consumption (men $<300 \mathrm{~g} /$ week and women $<150 \mathrm{~g} /$ week) in drinkers was the lower risk of CCV events compared with nondrinkers. About $70 \%$ of men drank less than $300 \mathrm{~g} /$ week and more than $90 \%$ of women drank less than $150 \mathrm{~g} /$ week. The authors concluded that alcohol intake showed J-shaped or U-shaped associations with the risk of CCV events in Japanese population.

The clinical course of diabetic nephropathy is classified into 5 stages: stage 1 hyperfiltration, stage 2 the silent stage, stage 3 microalbuminuria, stage 4 macroalbuminuria, and stage 5 uremia (23). In our study, we showed that DM correlated positively with a decrease in eGFR in PA patients. The reason for this correlation was that $75 \%$ of the participating patients had stage 1 to 3 diabetic nephropathy (eGFR; 76.8 [63.591.0] $\mathrm{mL} / \mathrm{min} / 1.73 \mathrm{~m}^{2}$, proteinuria; $24.8 \%$ ). When our patients were divided into 2 groups-the nonproteinuria group and proteinuria group-our analysis showed no correlation between DM and eGFR in the proteinuria group, whereas DM and eGFR correlated significantly with each other in the nonproteinuria group. One limitation of this study was its retrospective cross-sectional design. Further studies are needed to determine the effect of long-term treatment of PA patients on glucose intolerance, DM, and incidence of CCV events.

In conclusion, this is the first report to demonstrate the presence of DM as an independent risk factor for CCV events and renal complications even in PA patients. Management of DM should be considered in addition to the specific treatment of PA.

\section{Acknowledgments}

We wish to thank the JPAS/JRAS Study Group for collecting the clinical data.
Financial Support: This work was supported by the Japan Agency for Medical Research and Development (Grants JP17ek0109112 and JP19ek0109352); the National Center for Global Health and Medicine, Japan (Grants 27-1402 and 30-1008); and a Health Labour Sciences Research Grant (Nanchitou [Nan]-Ippan-046) from the Ministry of Health, Labor and Welfare, Japan.

\section{Additional Information}

Correspondence and Reprints: Michio Otsuki, MD, PhD, Department of Metabolic Medicine, Osaka University Graduate School of Medicine, 2-2 Yamada-oka, Suita, Osaka 565-0871, Japan. E-mail: otsuki@endmet.med.osaka-u.ac.jp.

Disclosure Summary: The authors have nothing to disclose.

Data Availability: The datasets generated during and/or analyzed during the current study are not publicly available but are available from the corresponding author on reasonable request.

\section{References}

1. Käyser SC, Dekkers T, Groenewoud HJ, et al. Study heterogeneity and estimation of prevalence of primary aldosteronism: a systematic review and meta-regression analysis. J Clin Endocrinol Metab. 2016;101(7):2826-2835.

2. Monticone S, D'Ascenzo F, Moretti C, et al. Cardiovascular events and target organ damage in primary aldosteronism compared with essential hypertension: a systematic review and metaanalysis. Lancet Diabetes Endocrinol. 2018;6(1):41-50.

3. Mulatero P, Monticone S, Bertello C, et al. Long-term cardio- and cerebrovascular events in patients with primary aldosteronism. $J$ Clin Endocrinol Metab. 2013;98(12):4826-4833.

4. Greene EL, Kren S, Hostetter TH. Role of aldosterone in the remnant kidney model in the rat. J Clin Invest. 1996;98(4):1063-1068.

5. Hollenberg NK. Aldosterone in the development and progression of renal injury. Kidney Int. 2004;66(1):1-9.

6. Rossi GP, Bernini G, Desideri G, et al; PAPY Study Participants. Renal damage in primary aldosteronism: results of the PAPY Study. Hypertension. 2006;48(2):232-238.

7. Pugliese G. Updating the natural history of diabetic nephropathy. Acta Diabetol. 2014;51(6):905-915.

8. Murase K, Nagaishi R, Takenoshita H, Nomiyama T, Akehi Y, Yanase T. Prevalence and clinical characteristics of primary aldosteronism in Japanese patients with type 2 diabetes mellitus and hypertension. Endocr J. 2013;60(8):967-976.

9. Fallo F, Veglio F, Bertello C, et al. Prevalence and characteristics of the metabolic syndrome in primary aldosteronism. J Clin Endocrinol Metab. 2006;91(2):454-459.

10. Reincke M, Meisinger C, Holle R, et al; Participants of the German Conn's Registry. Is primary aldosteronism associated with diabetes mellitus? Results of the German Conn's Registry. Horm Metab Res. 2010;42(6):435-439.

11. Remde H,Hanslik G, Rayes N, Quinkler M. Glucose metabolism in primary aldosteronism. Horm Metab Res. 2015;47(13):987-993.

12. American Diabetes Association. Diagnosis and classification of diabetes mellitus. Diabetes Care. 2010;33(Suppl 1):S62-S69.

13. Committee of the Japan Diabetes Society on the Diagnostic Criteria of Diabetes Mellitus. Report of the committee on the classification and diagnostic criteria of diabetes mellitus. J Diabetes Investig. 2010;1(5):212-228.

14. Nishikawa T, Omura M, Satoh F, et al; Task Force Committee on Primary Aldosteronism, The Japan Endocrine Society. Guidelines 
for the diagnosis and treatment of primary aldosteronism-the Japan Endocrine Society 2009. Endocr J. 2011;58(9):711-721.

15. Shimamoto K, Ando K, Fujita T, et al; Japanese Society of Hypertension Committee for Guidelines for the Management of Hypertension. The Japanese Society of Hypertension guidelines for the management of hypertension (JSH 2014). Hypertens Res. 2014;37(4):253-390.

16. Catena C, Colussi G, Nadalini E, et al. Cardiovascular outcomes in patients with primary aldosteronism after treatment. Arch Intern Med. 2008;168(1):80-85.

17. Hundemer GL, Curhan GC, Yozamp N, Wang M, Vaidya A. Cardiometabolic outcomes and mortality in medically treated primary aldosteronism: a retrospective cohort study. Lancet Diabetes Endocrinol. 2018;6(1):51-59.

18. Sechi LA, Novello M, Lapenna R, et al. Long-term renal outcomes in patients with primary aldosteronism. JAMA. 2006;295(22):2638-2645.

19. Akehi Y, Yanase T, Motonaga R, et al; Japan Primary Aldosteronism Study Group. High prevalence of diabetes in patients with primary aldosteronism (PA) associated with subclinical hypercortisolism and prediabetes more prevalent in bilateral than unilateral PA: a large, multicenter cohort study in Japan. Diabetes Care. 2019;42(5):938-945.

20. Ohashi K, Hayashi T, Watanabe Y, et al. Primary aldosteronism with type 2 diabetes mellitus requires more antihypertensive drugs for blood pressure control: a retrospective observational study. J Clin Med Res. 2018;10(1):56-62.

21. Ardisson Korat AV, Willett WC, Hu FB. Diet, lifestyle, and genetic risk factors for type 2 diabetes: a review from the Nurses' Health Study, Nurses' Health Study 2, and Health Professionals' Follow-Up Study. Curr Nutr Rep. 2014;3(4):345-354.

22. Saito $\mathrm{E}$, Inoue $\mathrm{M}$, Sawada $\mathrm{N}$, et al. Impact of alcohol intake and drinking patterns on mortality from all causes and major causes of death in a Japanese population. J Epidemiol. 2018;28(3):140-148.

23. Mogensen CE, Christensen CK, Vittinghus E. The stages in diabetic renal disease. With emphasis on the stage of incipient diabetic nephropathy. Diabetes. 1983;32(Suppl 2):64-78. 